\title{
¿Qué es una buena clase en ingeniería desde el punto de vista de los estudiantes?
}

\author{
Zadkiel Zuluaga-Rendón $^{a}$, Javier Alejandro Corredor ${ }^{a}$, Jesús María Quintero ${ }^{b}$, Jhon Jairo Ramírez-Echeverry ${ }^{b}$ \& \\ Fredy Andrés Olarte ${ }^{b}$
}

\begin{abstract}
${ }^{a}$ Facultad de Ciencias Humanas, Universidad Nacional de Colombia, Bogotá, Colombia.zzuluagar@unal.edu.co, jacorredora@unal.edu.co ${ }^{b}$ Facultad de Ingenierí, Universidad Nacional de Colombia, Bogotá, Colombia.jmquinteroqu@unal.edu.co, jjramireze@unal.edu.co,faolarted@unal.edu.co
\end{abstract}

\begin{abstract}
Resumen - La realización de este estudio responde a la necesidad de diseñar ambientes académicos que mejoren la adquisición de los conceptos en ingeniería que resultan a veces abstractos y limitan su comprensión. Utilizando metodologías cualitativas, se explora cuáles son los principales factores que los estudiantes refieren cuando evalúan las clases de ingeniería con el propósito de establecer elementos comunes entre las clases consideradas como destacadas. Para tal fin, las evaluaciones abiertas realizadas a profesores de ingeniería de la Universidad Nacional de Colombia fueron codificadas y analizadas para encontrar los temas subyacentes a categorías previamente identificadas en la literatura psicológica. Adicionalmente, se compararon los conteos de frecuencias en estos factores entre los profesores ubicados en el primer y último grupo de la evaluación cuantitativa. Los resultados mostraron que los estudiantes consideraban, dentro de la categoría de metodología, factores asociados a la evaluación, a la dinámica de clase y a la participación, y a la organización de tiempos y espacios. Finalmente, dentro de la categoría de huella docente, los estudiantes incluyeron la dedicación y el compromiso del docente, el nivel de asertividad, y la motivación y actitud. Todos estos factores diferenciaban el primer del último grupo.
\end{abstract}

Palabras clave: estrategias pedagógicas, educación en ingeniería, identidad, análisis Cualitativo, metodología de enseñanza; experticia.

Recibido: 8 de octubre de 2016. Revisado: 5 de noviembre de 2016. Aceptado: 15 de diciembre de 2016.

\section{What makes a good class from the students' point of view?}

\begin{abstract}
This study seeks to provide an answer to the need to design better academic environments in order to improve conceptual acquisition in abstract engineering concepts. It explores the main factors affecting engineering professors' evaluations using qualitative methodologies. In order to do so, the open questions answered by students when assessing professors at Universidad Nacional de Colombia were coded and analyzed to identify the topics that underlie categories that were identified from prior educational literature. Additionally, professors who obtained quantitative evaluations in the first and fourth quartile were compared in terms of the code frequencies that they obtained in qualitative evaluations. Results showed that students considered, within the category of teaching methods, elements related to assessment, class dynamics and participations, and the organization of spaces and time within the class. Finally, within the category of the professor's footprint, students included dedication and commitment to the class, his or her assertiveness, and his or her motivation and attitude when teaching. All these factors differentiated teachers in the first and fourth quartile.
\end{abstract}

Keywords: pedagogical strategies, engineering education, identity, qualitative analysis, teaching methods, expertise.

\section{Introducción}

Este artículo hace parte de la agenda de investigación del Centro de Innovación Educativa Regional-Región Centro, de la Universidad Nacional de Colombia.

Una clase de ingeniería depende, entre otros, de factores como el ambiente, los recursos, las estrategias didácticas y los mecanismos de evaluación para un logro efectivo de los objetivos de formación. No obstante, un factor determinante en la clase es el profesor, quien define y desarrolla las actividades asociadas al desarrollo de su asignatura para que los estudiantes puedan generar los conocimientos o habilidades esperados. Es por esto que la evaluación de las actividades docentes, tanto a nivel metodológico como profesional y empático, realizada por parte de los estudiantes es un insumo fundamental para la implementación de acciones de mejoramiento en el ambiente universitario.

Experiencias en contextos educativos superiores, han mostrado como la triangulación de evaluaciones por pares académicos e instrumentos cualitativos (e.g., rúbricas, portafolios, test de desempeño, etc.), con evaluaciones a estudiantes, inciden en la construcción de un Feedback efectivo y el mejoramiento del desempeño docente [1-4]. Este tipo de evaluaciones es ampliamente utilizado en programas de formación docente en general. Con respecto a experiencias locales en cuanto a clases de ingeniería, se ha usado la entrevista a estudiantes como parte de un modelo integral de evaluación del desempeño docente en cuanto a eficiencia y calidad percibida [5], aunque con ciertas limitaciones. Ya que la herramienta utilizada en esta evaluación genera un índice estadístico, el tipo de entrevista es estructurada y consiste en la puntuación de cierta cantidad de ítems prediseñados en una escala tipo Likert. Si bien este tipo de pruebas se realizan con la mayor rigurosidad estadística para garantizar la precisión de sus inferencias, deja de lado la posibilidad de explorar otros factores que pueden estar influenciando la percepción del desempeño y calidad docente.

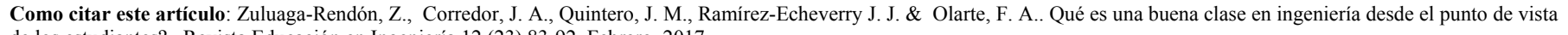
de los estudiantes? . Revista Educación en Ingeniería 12 (23) 83-92, Febrero, 2017. 
Con el fin de determinar posibles campos de mejoramiento de las actividades docentes, la Universidad Nacional de Colombia lleva a cabo la evaluación Edificando. Esta evaluación califica tres aspectos de los docentes desde la percepción de los estudiantes quienes tomaron clases con ellos en el periodo académico correspondiente. Estos tres factores son: 1. Empatía con el profesor o 'Huella'; 2. Fomento del pensamiento crítico y autonomía, motivación por los temas; y 3. Preparación y agrado dictando clase, respeto y adaptación a las necesidades del grupo, imparcialidad y pertinencia de las evaluaciones. Los anteriores factores se evalúan cuantitativamente lo que permite obtener un valor numérico de desempeño global de los docentes. Adicionalmente, el Edificando cuenta con dos espacios de respuesta abierta para realimentación cualitativa. Si bien obtener puntuaciones numéricas para los tres factores mencionados permite tener una noción sobre la percepción de los estudiantes acerca del desempeño docente, no reconoce las actividades específicas que éstos llevan a cabo en el desarrollo de las clases y que además podrían ser insumos guiados para el mejoramiento de las clases.

Este artículo presenta una aproximación a responder la pregunta: ¿Qué aspectos del desempeño docente construyen una buena clase en ingeniería desde el punto de vista de los estudiantes? utilizando la información de los dos ítems abiertos de la evaluación Edificando. Los datos de la evaluación docente se categorizaron para identificar qué aspectos específicos de la práctica docente al interior de las clases son considerados relevantes, desde el punto de vista de los estudiantes.

En la construcción de una buena clase no se trata de asumir la opinión de los estudiantes ciegamente, sino de entender cómo comentarios de diverso tipo, se relacionan con conceptos fundamentales de la psicología educativa y la pedagogía contemporáneas y cómo estos se observan en las actividades específicas de las clases de ingeniería. A continuación, se presenta una corta revisión teórica de los principales conceptos mencionados en la literatura al respecto.

\subsection{Rutinas}

Un concepto clave en el éxito de una asignatura es la construcción de rutinas [6,7]. Rutina se refiere acá a una secuencia de acciones que cumple un fin pedagógico determinado y que se repite consistentemente dentro de un curso $o$ asignatura [8]. Las rutinas hacen predecible la clase para los estudiantes y descargan recursos cognitivos de lo logístico, para concentrarse en aspectos de contenido. En otras palabras, las rutinas permiten que los estudiantes concentren sus esfuerzos en la comprensión del tema, y no en la predicción de las acciones del docente. Esto es así, porque las rutinas ordenan la clase y le dan estructura en función de objetivos pedagógicos. También, un docente con un conjunto de rutinas variado tiene una mayor capacidad para hacer una clase entretenida, y, al mismo tiempo, rigurosa. Esto es así, porque las rutinas se asocian a acciones pedagógicas [9]. Por ejemplo, un docente en una clase de matemáticas puede tener tres tipos de rutinas. Una parte asociada a la exposición de los elementos conceptuales que subyacen los procedimientos; una segunda parte asociada a la explicación de procedimientos y algoritmos; y, finalmente, una tercera parte enfocada en la identificación de errores en procedimientos, bien sea dentro de los ejercicios realizados por los estudiantes, como en casos preparados por los estudiantes. Estas tres rutinas, repetidas sostenidamente, durante un curso, hacen que los estudiantes puedan predecir la estructura de la clase, al mismo tiempo que desarrollar las habilidades en las que se enfocan cada una de estas rutinas (e.g., identificar errores en ciertos procedimientos matemáticos).

\subsubsection{Tipos de tareas}

Un segundo concepto que necesita ser revisado es el de tipos de tareas. En la literatura psicológica se distinguen dos tipos fundamentales de tareas: las tareas abiertas y cerradas [10]. Una tarea abierta es una tarea en la cual los estados ideales de solución y las operaciones no son fijos. Estas tareas son conocidas, como tareas mal definidas (ill-defined). Una tarea cerrada es, por ejemplo, un ejercicio matemático con una única respuesta y un camino de solución, o varios caminos, pero dentro de un conjunto de opciones limitado. Una tarea abierta es un desarrollo tecnológico o un proyecto de investigación. La diferencia es que, en el primer caso, la solución del problema pasa por encontrar el camino de respuesta y la respuesta correcta, lo que dado que los estados son definibles se puede realizar algorítmicamente (por ejemplo, evaluando la discrepancia entre estados ideales y estados actuales) [11]. Una tarea abierta, por el contrario, requiere que el solucionador construya el espacio de tarea llenando vacíos en un espacio muy amplio de opciones [12]. Esta connotación, sin embargo, no tiene connotaciones valorativas, es decir, las tareas mal definidas no son consideradas inferiores a las tareas bien definidas de ninguna manera dentro de la literatura educativa. Por el contrario, las tareas abiertas son consideradas ideales para ciertos tipos de aprendizaje. En el otro lado del espectro, existen las tareas cerradas cuyos estados finales y operaciones están definidos de antemano [13].

En términos pedagógicos, la diferencia entre estos tipos de tareas es clave porque un buen proceso pedagógico necesita combinar ambos tipos de tareas, pero también porque las estrategias pedagógicas más efectivas son diferentes para cada tipo. Es decir, lo que es efectivo en términos pedagógicos para las tareas abiertas, como la elaboración de explicaciones teóricas, no lo es necesariamente para las tareas cerradas, como por ejemplo el control de variables [14]. Por ejemplo, las prácticas de instrucción directa, donde el docente asume el rol de transmisor de información, son más efectivas en tiempos cortos de instrucción, mientras que procesos de aprendizaje por descubrimiento guiado son más efectivos en tiempos instruccionales más largos [15].

El punto fundamental es que la investigación pedagógica ha mostrado que la enseñanza tiene diferentes parámetros para cada tipo de tarea [16]. Por ejemplo, la enseñanza de tareas cerradas se puede hacer a través de instrucción directa y modelamiento, para reducir la carga cognitiva de los estudiantes y hacer más efectivo el proceso de aprendizaje. Esto, en la práctica, implica que, en tareas cerradas (e.g., despejar ecuaciones), no requiere que los estudiantes descubran las estrategias, sino que estas pueden ser modeladas por los docentes. Las tareas abiertas, por el contrario, tienen otros 
requerimientos cognitivos y pedagógicos. Esto es así, porque la capacidad de resolver tareas abiertas pasa por una exposición a la complejidad que los contextos reales, profesionales y de investigación requieren [16]. En este caso, la estrategia pedagógica más adecuada es el modelamiento guiado. Esto quiere decir que el docente debe plantear tareas abiertas (e.g., como resolver un problema de diseño), y acompañar a los estudiantes a través de preguntas y sugerencia de conceptos en el diseño. En este caso, la clave es que el docente genere incertidumbre, pero también acompañe a los estudiantes en la tarea.

\subsection{Transferencia}

La razón por la cual la buena enseñanza requiere combinar tareas abiertas y cerradas es que la investigación ha mostrado que no existe transferencia entre ambos ambientes de trabajo [17]. Estudiantes que han sido acostumbrados sólo a resolver tareas cerradas, rara vez son capaces de utilizar esos conceptos en una tarea abierta, por ejemplo, un proyecto. En ese sentido, es importante señalar el problema de los isomorfos que es un problema clave en el estudio de la transferencia [18]. Fundamentalmente, el problema de los isomorfos se refiere al hecho de que poseer el conocimiento, declarativo o procedural, necesario para resolver un problema, no es garantía de que un estudiante sea capaz de aplicarlo al contexto correcto. En investigación básica en psicología, se ha visto que sujetos con el conocimiento necesario para resolver una tarea son incapaces de resolver tareas isomorfas (similares), en términos de la descripción lógica de la tarea, pero diferentes en elementos superficiales. En otras palabras, las personas son reactivas a los elementos superficiales en la presentación de una tarea porque esos elementos determinan la capacidad de conectar su conocimiento acumulado, y aplicarlo efectivamente a la resolución de problemas. En este sentido, una educación efectiva requiere modelar el reconocimiento de isomorfos, más allá de elementos superficiales. Esta tarea, sin embargo, toma formas distintas cuando se trata de tareas abiertas y tareas cerradas. Para el caso de las tareas cerradas, la mejor ruta es la instrucción directa que señale estructuras similares, en ejemplos que varían en rasgos superficiales (e.g., cuando se señala una misma estructura matemática en diferentes ecuaciones), acompañada de ejercicios prácticos que respalden el afianzamiento de lo enseñado [19]. Para el caso de las tareas abiertas, la mejor ruta es el acompañamiento constante del tutor o docente en un proceso de continuo andamiaje del aprendizaje que permita conectar los retos de diseño e investigación, con los conceptos teóricos relevantes [20].

\subsection{Experticia}

Un concepto particularmente importante para entender cómo se deben realizar estos modelamientos es el concepto de experticia. La experticia, entendida desde la literatura psicológica, refiere a las características de los procesos de razonamiento y resolución de problemas de los sujetos con altos niveles de desempeño en un área determinada [21]. Se ha encontrado, a través de investigación diversa, que este desempeño no depende de un talento innato o de una capacidad especial, sino de un entrenamiento sostenido a lo largo de mucho tiempo, diez años como mínimo [22]. En particular, la investigación ha encontrado que existen similitudes entre expertos en áreas tan diversas como la música y la física, que parecen señalar ciertas características como claves en el desarrollo del alto desempeño [23].

Una característica que se ha encontrado consistentemente es la capacidad de distinguir rasgos profundos y superficiales en la resolución de problemas. Mientras los expertos enfocan sus procesos de resolución de problemas en principios centrales del dominio de conocimiento, los novatos parecen concentrarse en rasgos superficiales. Por ejemplo, se ha encontrado que los físicos expertos categorizan problemas de física y organizan sus estrategias de resolución en función de los principios de la mecánica (para el caso de problemas en esta área), mientras los novatos lo hacen enfocándose en rasgos superficiales, como la presencia de ciertas formas geométricas en los diagramas [24]. Esta diferencia en la percepción de rasgos superficiales y profundos es central a la comprensión de la experticia, y ha sido replicada en áreas tan diversas como la medicina, la música, la psicología, y las ciencias naturales [25]. Esta distinción es importante para la enseñanza porque el docente es un experto en el área y en este sentido debería concentrarse en transmitir la estructura del dominio $\mathrm{y}$, particularmente, la distinción entre rasgos superficiales y profundos a sus estudiantes. En este sentido, se requiere que el docente sea capaz de resaltar los aspectos profundos de la disciplina, y diferenciarlos de los aspectos superficiales de ésta. Esta distinción debe promoverse porque una de las razones por las que los expertos son superiores a los novatos, no es que tengan una capacidad mental superior sino porque piensan en unidades más grandes y se enfocan en aspectos relevantes, desperdiciando menos recursos cognitivos en aspectos secundarios. En este sentido, el modelamiento activo del docente, conectando, por ejemplo, diferentes problemas o conceptos a un núcleo central en el área es un proceso fundamental en el desarrollo del pensamiento experto en el estudiante.

Otra implicación importante para la enseñanza es que el experto debe aplicar un plan de entrenamiento flexible con ejemplos diversos que varíen en sus rasgos superficiales y sus características isomorfas. Esto es, el docente debe preparar ejemplos que sistemáticamente exploren variaciones en los problemas para mostrar a los estudiantes qué elementos son esenciales, y cuales no lo son, en la resolución de un tipo de tarea dado. La variedad en las características de los problemas y situaciones, se justifica además porque la investigación ha mostrado que el logro de la experticia no surge de un entrenamiento repetitivo, sino de un entrenamiento variado, denominado práctica deliberada, que obliga al aprendiz a salir de la zona de confort. Tanto en tareas abiertas como en tareas cerradas, el experto debe proponer situaciones novedosas que reten a los estudiantes, y modelar o acompañar, según sea el caso, en la solución de estas.

\subsection{Identidad y huella docente}

Un último concepto que debe ser explorado es el de identidad o huella docente. Este concepto se refiere a cambios que suceden en el estudiante más allá de lo cognitivo y que se asocian a ganancias en términos afectivos, en su construcción como sujetos $\mathrm{y}$ en su relación emocional con los contenidos. La idea de 
identidad surge en la literatura psicológica cuando los investigadores empiezan a encontrar que incluso cuando la enseñanza se enfoca exclusivamente en contenidos, ésta produce cambios en los elementos mencionados anteriormente. Por ejemplo, [26] han mostrado que prácticas educativas verticales producen identidades en las que los sujetos consideran que su rol epistemológico es exclusivamente el de receptores, y no el de productores de conocimiento. Esto tiene mucho sentido si se tiene en cuenta que los estudiantes sometidos a prácticas verticales de enseñanza deben exclusivamente aprender y repetir lo que el docente está predicando. En este sentido, el docente es un constructor de algo más que contenidos: es un modelo de rol que prefigura lo que un profesional en un área debe ser. En este sentido, la práctica docente debería crear en el estudiante prerrequisitos importantes para la investigación e intervención de alto rendimiento, como la pasión por el conocimiento y las formas de relacionarse con otras personas dentro y fuera del grupo. Entre los resultados más relevantes en investigación alrededor de la identidad se cuenta el hecho de que los estudiantes en el aula no sólo aprenden conocimientos y métodos de resolución de problemas, sino también características de las comunidades de práctica a las que se enfrentarán durante su desempeño profesional [27], y el descubrimiento de que ciertos tipos de enseñanza favorecen la construcción de roles que se asocian a las características implícitas en estas prácticas [28].

Por esto, cada docente debe pensar muy cuidadosamente qué tipo de estudiante pretende formar. Prácticas, como la excesiva presión durante las tareas de resolución de problemas o en las prácticas de aula tienen más sentido en ciertas profesiones que en otras. Por ejemplo, si se están educando médicos, la capacidad de trabajar bajo presión es clave para el desarrollo profesional y puede tener sentido presionar a los estudiantes durante estas tareas. En otras áreas, como por ejemplo la ingeniería, este tipo de modelamiento puede ser contraproducente, ya que afecta el desempeño en prácticas importantes como el trabajo en equipo y la ejecución colaborativa de proyectos. Más allá de esto, un docente que es apasionado por su área de investigación, trasmite y crea investigadores, particularmente durante los años formativos de la universidad, que son claves en el desarrollo identitario. Un docente apático, o peor, resentido con su desarrollo profesional, transmite también el sentido de que ser profesional en el área es una obligación externa, sin ninguna motivación personal.

Estos precedentes teóricos señalan que existe una literatura amplia sobre lo que puede prefigurar una buena clase. Una cuestión abierta es, sin embargo, si los estudiantes universitarios de ingeniería son reactivos a estos factores a la hora de calificar una clase. Esta investigación revisa esta cuestión, a partir de una consideración cuidadosa de los aspectos que los estudiantes consideran relevantes dentro de evaluaciones. Para esto se realizó la investigación cualitativa que se describe a continuación y que buscaba responder esta pregunta: ¿Qué aspectos del desempeño docente construyen una buena clase en ingeniería desde el punto de vista de los estudiantes?

\section{Método}

Para evaluar el desempeño docente a partir de la experiencia de los estudiantes, esto es, determinando los factores que para los estudiantes constituyen una buena clase, se analizaron los resultados del programa Edificando de la Universidad Nacional de Colombia para el Departamento de Ingeniería Eléctrica y Electrónica (DIEE). Este programa realiza mediciones periódicas de los docentes que abarcan evaluaciones cuantitativas de las clases, y evaluaciones cualitativas abiertas de estas. En esta investigación, se realizó un análisis de contenido a las respuestas abiertas del Edificando para los últimos cuatro periodos académicos. En este se construyeron categorías que organizaban las respuestas de los estudiantes, posteriormente se cruzaron los puntajes numéricos asignados a cada profesor con las frecuencias de aparición de estas categorías para establecer que características diferenciaban a los profesores ubicados en el cuartil más alto de aquellos ubicados en el cuartil más bajo.

\subsection{Participantes}

Se analizaron las evaluaciones del programa Edificando de los profesores vinculados al departamento de Ingeniería Eléctrica y Electrónica que contaban con participación en por lo menos el último periodo académico al momento de iniciar el proceso; esto es, quienes contaban con evaluaciones entre los periodos 2013-II y 2015-I y habían tenido clases a su cargo en el periodo de 2015-I. Fueron en total 39 docentes ( 38 hombres y una mujer). Esta distribución por género es en alguna manera inevitable dada la distribución de los programas de ingeniería.

\subsection{Análisis programa edificando DIEE}

El programa de evaluación docente consta de 19 ítems cerrados y dos abiertos, estos últimos, indagan por los aspectos a destacar y aspectos por mejorar en el desarrollo de la clase y la práctica docente de cada educador. A partir de estos dos últimos ítems se realizó el proceso de codificación. Para codificar las participaciones de los estudiantes en el programa, se elaboraron tres categorías construidas a partir de coincidencias y cercanías conceptuales con la teoría revisada. Esta codificación se realizó también atendiendo al hecho de que las categorías utilizadas dieran cuenta de la diversidad de respuestas producidas por los estudiantes. De hecho, diversos sistemas de codificación fueron contrastados frente a los datos, y categorías más pequeñas que emergieron también fueron incluidas en los análisis. En otras palabras, el análisis de contenido se realizó combinando una codificación estructurada basada en hipótesis $[29,30]$ y una codificación atendiendo a temas emergentes. Esta última codificación se basó en la tradición más clásica de la teoría fundamentada [31]. Es importante notar, sin embargo, que se realizó la identificación emergente de categorías, pero no los niveles de codificación axial o selectiva, ya que no eran necesarios para la identificación básica de temas que tenía como objetivo este estudio. Las categorías elegidas inicialmente para la codificación fueron metodología, dominio y experticia, y huella docente. Además de la codificación en estas categorías, se tuvo en cuenta la valencia de las interacciones, positiva o negativa, lo cual permitió realizar la calificación global del desempeño a partir del punto de vista de sus estudiantes. Adicionalmente se 
identificaron categorías emergentes que le dan forma y configuran los elementos que constituyen las categorías previstas desde la revisión teórica. Es importante anotar también que cada interacción podía ser ubicada en más de una categoría siempre y cuando refiriera factores que se encontraran en más de una categoría.

Los resultados de estas codificaciones se cruzaron con los resultados de la evaluación cuantitativa de los docentes para ver, por un lado, cuál era el nivel de validez predictiva de la codificación, y, por el otro, si existía una relación entre las prácticas pedagógicas que emergieron en la codificación y la opinión de los estudiantes sobre la clase. Estos análisis nos permiten realizar un análisis más profundo con respecto a acciones específicas que los docentes llevan a cabo dentro del aula de clase y en su proceso pedagógico en general. A continuación, se describen las categorías con sus correspondientes factores y también la relación de estas con los resultados cuantitativos. La codificación se presenta en la sección de resultados porque si bien las categorías macro metodología, dominio y experticia y huella docente- se habían establecido de antemano, las subcategorías que las describen fueron identificadas dentro del análisis cualitativo.

\section{Resultados}

Como se indicó con anterioridad, este estudio identifica una serie de temas que subyacen tres categorías teóricas extraídas de la literatura previa: metodología, dominio y experticia y huella docente. Como se explicó con anterioridad, se realizó una identificación de temas emergentes que daban cuenta de los aspectos que eran relevantes para cada uno de estos factores. Estos códigos fueron revisados, reconstruidos y recodificados hasta considerar que daban cuenta de la complejidad de los datos observados cumpliendo con los criterios de saturación por cada subcategoría, estas deberían aparecer en por lo menos el $10 \%$ del total de los docentes evaluados en este periodo. Esto es, sólo se mantuvieron códigos que aparecieran reiterativamente dentro de la muestra de evaluaciones; es decir, que las referencias a los códigos emergentes fueran realizadas en diferentes evaluaciones [32]. En términos, cuantitativos $56 \%$ de los resultados mostraron referencias a las categorías de metodología, 18\% hicieron referencia a asuntos de dominio y experticia y el $26 \%$ se relacionaron con elementos de la huella docente.

\subsection{Metodología}

Las interacciones en esta categoría relacionan todas las acciones que lleva a cabo el docente en el desarrollo de las clases para lograr el objetivo pedagógico del curso, la enseñanza y el aprendizaje de los contenidos temáticos propuestos en el desarrollo del mismo. La metodología que el docente desarrolla en su clase, define la manera transferencial de las tareas con respecto a lo aprendido en el aula y el contexto de desempeño real en la ingeniería en cuanto a lo asertividad de las tareas, abiertas o cerradas, que hacen parte de su modelo pedagógico. A partir de la codificación emergente esta categoría se dividió en los siguientes seis factores:

\subsubsection{Evaluación - Calificación}

Este factor se calificó cuando la opinión del estudiante se refería al proceso de evaluación y calificación de los contenidos vistos en clase realizados por el docente. Entre los aspectos que se mencionaban comúnmente en esta categoría estaban la calificación de trabajos, parciales, adelantos, fechas de entrega entre otros.

Es muy justo con todas las notas, califica con respecto al trabajo que uno ha llevado a cabo durante el semestre. Evaluar más seguidos los temas y sobre los ejercicios realizados en clase, pues una sola evaluación y trabajo no equivalen a la evaluación de todo un curso.

\subsubsection{Dinámica de la clase y participación del estudiante (herramientas)}

Una opinión califica en este factor siempre y cuando se refiera a actividades y estrategias que hacen o no agradables las sesiones de la clase, el uso de herramientas ya sean físicas, conceptuales o informáticas, y/o la participación del estudiante en el desarrollo de la clase (e.g. exposiciones de estudiantes).

Lleva una clase amena y con temas de gran interés, se preocupa por el aprendizaje de cada estudiante $y$ profundiza de acuerdo a los temas planteados.

Al menos en dos o tres clases, explicar los temas más importantes del ***** con ayuda de herramientas visuales (proyector).

\subsubsection{Retroalimentación y explicación del tema (uso de ejemplos y talleres)}

Las opiniones se codificaron en este factor cuando se hacían alusión a la explicación de los temas desarrollados en la clase, el uso de ejemplos, talleres en clase para afianzar los conceptos teóricos y/o prácticos y la profundidad teórica con que se perciben.

La forma de explicar, de manera sencilla y muy eficaz cumpliendo con todos los temas propuestos, además se esmeró por que los estudiantes aprendiéramos y nos interesáramos por las temáticas de la materia.

Explicar más sobre los ******, y no dar por sobreentendido algunos temas que él creía obvios.

\subsubsection{Uso de analogías/aplicaciones en contextos reales- cotidianos}

Un comentario registrado en este factor refleja una opinión respecto a la explicación y ejemplificación de los temas desarrollados en clase con analogías o contextos reales de aplicación o trabajo.

El docente compartía constantemente trabajos actuales desarrollados por él, como material de estudio.

Salir un poco de los libros y mostrar un poco más los problemas reales.

\subsubsection{Organización, disposición del tiempo y espacios de clase}

Las opiniones ubicadas en este factor, dan cuenta de la percepción de la organización de la clase, la optimización del 
tiempo de la misma y el uso de los espacios del aula, laboratorios, espacios de formación externos al campus y disposición de tiempos extra clase para asesorías y/o tutorías.

El poder ir a ***** es una gran ventaja ya que aprendemos y vemos realmente como se aplican y cuáles son las herramientas que saldremos a utilizar en nuestro diario como ingenieros.

Los laboratorios más extensos pudieron ser divididos en unos más pequeños más alcanzables.

\subsubsection{Acompañamiento y aprendizaje autónomo del estudiante}

Aquí se encuentran las opiniones de los estudiantes referentes a los procesos de aprendizaje autónomo promovidos por los profesores, como se perciben y si el proceso de enseñanza está siendo o no acompañado y orientado por parte del docente.

Más que dar respuesta a nuestras dudas nos ayudó a que nosotros mismos diéramos esas respuestas siempre con su orientación para no llegar a conclusiones erróneas.

Su metodología no es la mejor de todas, el aprendizaje autónomo es importante, pero eso no reemplaza la enseñanza de un profesor y es en parte lo que hace, de resto todo bien.

Los resultados de las codificaciones realizadas muestran que la metodología no es un constructo unitario, sino que por el contrario se divide en varios subfactores. Estos subfactores indican de buena manera las estrategias que pueden utilizar los docentes para mejorar su práctica pedagógica. Algunos de estos aspectos son la introducción de analogías y ejemplos, la presencia de retroalimentación, un uso adecuado del tiempo, y un balance adecuado entre trabajo autónomo y guía. Sin embargo, cuando la codificación realizada fue comparada entre el grupo de mejor $(\mathrm{G} 1)$ y peor (G4) calificación en la evaluación cuantitativa, se encontró que sólo los factores de evaluación, dinámica y participación, y organización de tiempos y espacios, predecían los resultados de los docentes en las evaluaciones cuantitativas (Fig. 1). Los subgrupos G1 y G4 se seleccionaron del grupo Total, usando los cuartiles superior e inferior respectivamente. Los valores obtenidos del índice para esta comparación se obtuvieron restando los comentarios con valencia negativa de los comentarios con valencia positiva, y se dividía por el total de comentarios. Este índice se ajustó para que tuviera un rango entre 0 y 1 para facilitar la presentación.

\subsection{Dominio y experticia}

En esta categoría, se ubican las opiniones que hacen referencia a los conocimientos por parte del profesor en el área profesional y académica, y a la forma en que los estudiantes perciben dicha capacidad. Las opiniones en esta categoría conciernen a la experiencia percibida, manejo teórico y experticia, ya sea como profesional o como pedagogo. Esta categoría se divide en los siguientes dos factores.

\subsubsection{Experiencia}

Las opiniones que conforman este factor, dan cuenta de la valoración por parte de los estudiantes de la experiencia y

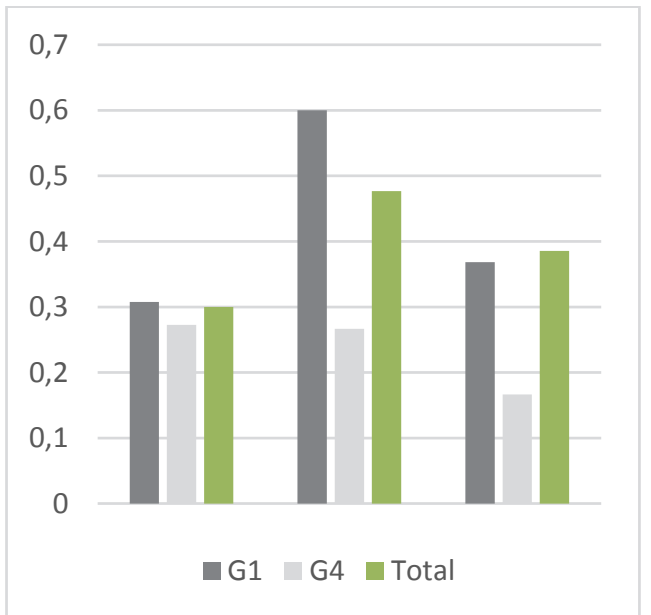

Figura 1. Factores de a) Evaluación - calificación, b) Dinámica - participación y c) Organización de tiempo y espacio, respectivamente, que discriminan entre los G1 y G4 en términos de metodología.

Fuente: Los autores.

habilidades adquiridas ya sea en la docente o profesional. Estos comentarios, en alguna medida, expresan la percepción que los estudiantes tienen de la experiencia efectiva del docente, $\mathrm{o}$, por lo menos, de la que transmite dentro del aula. Poseer docentes con experiencia y mantenerlos vinculados a los programas de formación en pregrado parece ser una derivada inmediata de este punto.

La preparación, experiencia y el conocimiento que evidenciaba en cada sesión de clase.

Creo que le hace falta experiencia con la clase de *****, no dudo que en el resto de materias sea un excelente profesor pero en esta no tenía ni idea de lo que estaba haciendo aunque se notaba que manejaba los temas no se hacía entender, y un profesor que no sepa enseñar creo que no debería enseñar.

\subsubsection{Dominio de tema}

Para ser ubicadas en este factor las opiniones deben dar cuenta del manejo de los temas y conceptos expuestos en clase y la fluidez con que estos son expuestos por parte del profesor. Este código, a diferencia del anterior, se relaciona más con el aspecto disciplinar de los cursos y menos con la experiencia profesional en el área. En ese sentido, posicionar a los profesores en temas que les sean conocidos, y mantenerlos durante varios semestres en los mismos cursos, puede ayudar a mejorar dicha percepción.

Su claridad sobre el tema en general de ***** y sobre los mismos *****. Su capacidad de transmitir sus conocimientos a los estudiantes.

Manejar mejor el tema, no por falta de conocimiento del mismo, sino de expresividad para hacerse entender. Los resultados de la codificación muestran que la percepción de dominio y experticia se relacionan, por un lado, con la experiencia previa percibida en el área, y, por el otro, con la una percepción de suficiencia en relación con el conocimiento disciplinar. Este factor, si se miran los fragmentos señalados en este texto, parece estar separado de la capacidad de trasmitir el 


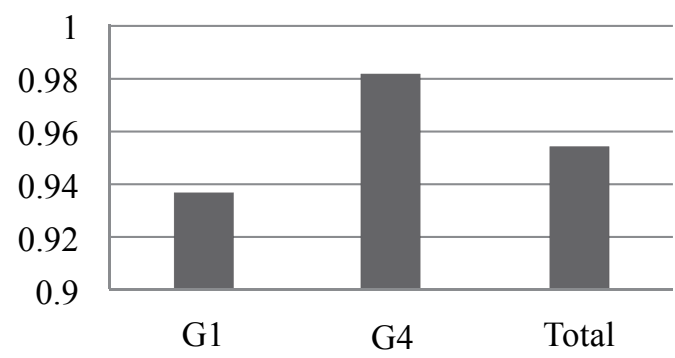

Figura 2. Diferencias en dominio y experticia entre G1 y G4 Fuente: Los autores.

contenido. Es decir, un docente puede demostrar un alto nivel de dominio y experticia, y, sin embargo, crear la sensación de que no es capaz de transmitir este conocimiento. De hecho, al revisar las diferencias entre los docentes que se encuentran en el grupo superior (G1) y los que se encuentran en el grupo inferior (G4) se encuentra que las codificaciones en dominio y experticia no favorecen a los docentes mejor calificados (Fig. 2 ). Lo que esto parece sugerir es que no basta con ser percibido como competente en términos de contenido y desempeño profesional, sino que se requiere trabajar en aspectos pedagógicos, de comunicación, de acompañamiento y de diseño instruccional para ser considerado un buen profesor. Aunque no se muestra en la Fig. 2, el patrón observado para la categoría general es muy similar para los dos factores que componen la categoría.

\subsection{Huella docente}

La categoría de huella docente reúne todas las opiniones relacionadas con las cualidades o actitudes propias del docente más allá de su labor metodológica, factores que intervienen en la relación pedagógica y en la construcción del ambiente de aprendizaje en el aula. Huella docente se divide en los siguientes cuatro factores que fueron identificados a partir de la codificación de los datos. Estos factores son dedicación y compromiso, responsabilidad y labor docente, asertividad comunicativa y respeto, y motivación y actitud en clase. Estos factores representan una percepción más amplia del docente más allá de su capacidad para transmitir contenidos: la clase de persona que transmite que es, su relación con el trabajo y con el área disciplinar que enseña.

\subsubsection{Dedicación y compromiso con el aprendizaje del estudiante}

En este factor, las opiniones hacen referencia al compromiso, dedicación y esfuerzo por parte del docente en el desarrollo de las clases. También se ubican en este, las opiniones que califican al docente a nivel personal o profesional. Definido en un sentido amplio, este factor describe la preocupación que el docente tiene por el aprendizaje de sus estudiantes, lo que en una medida amplia puede influir en la motivación de estos.

La preocupación por que el estudiante aprendiera no solo los temas vistos en clases sino temas que se utilizan fuera del aula de clase.

Este profesor no desempeña con agrado su labor docente, según nos comentó porque tenía cosas más importantes que hacer.

\subsubsection{Responsabilidad con la clase y labor docente (puntualidad)}

Este conjunto de opiniones hace referencia a la responsabilidad que el docente demuestra en el desarrollo de la clase y con los contenidos propuestos. Es frecuente encontrar alusiones a la puntualidad/impuntualidad o la presencia de otras actividades del docente que interfieren en el desarrollo de la clase. Un docente que prepara y cumple unos mínimos en su tarea docente, es un docente que transmite un sentido de responsabilidad, lo que a su vez le otorga legitimidad para exigir a los estudiantes.

La preparación del material de clase y las tareas. La puntualidad El conocimiento del tema.

El profesor es extremadamente impuntual, perezoso y despreocupado. Buena persona, seguramente buen ingeniero, pero como docente no es lo que esperaba.

\subsubsection{Asertividad comunicativa, respeto y parcialidad/imparcialidad con el estudiante}

Estas interacciones recogen la percepción sobre la calidad de la comunicación, el respeto en el diálogo docente-estudiante y la disposición del docente para recibir recomendaciones de los estudiantes. En algunos casos, docentes con buen dominio del contenido y con responsabilidad en relación con la clase, son percibidos como irrespetuosos o arrogantes en el trato a los estudiantes. Aunque, de nuevo, este factor no está relacionado con el aprendizaje en sí, sí tiene fuertes consecuencias sobre la motivación de los estudiantes. En un sentido más amplio, este factor es importante porque el docente es un modelo de rol que determina que tipo de profesional, y de persona, los estudiantes van a ser.

Su cordialidad y su forma amable de tratar a los estudiantes, su humildad y sin duda su gran conocimiento hacen del profesor ***** un gran docente.

Tolerancia con los estudiantes, humildad con el conocimiento y respeto hacia quienes lo rodean.

\subsubsection{Motivación y actitud en clase}

Las opiniones incluidas en este factor hacen referencia a la labor o cualidades del docente que animan o desaniman al estudiante en el aprendizaje de los temas desarrollados en clase, o por la carrera misma. El factor refiere elementos asociados al entusiasmo y la disposición que el docente transmite en relación con el tema a enseñar, $y$, en general, con la actividad académica y profesional. Un docente que transmite una baja motivación hacia el dominio y el aprendizaje difícilmente podrá llevar a sus 


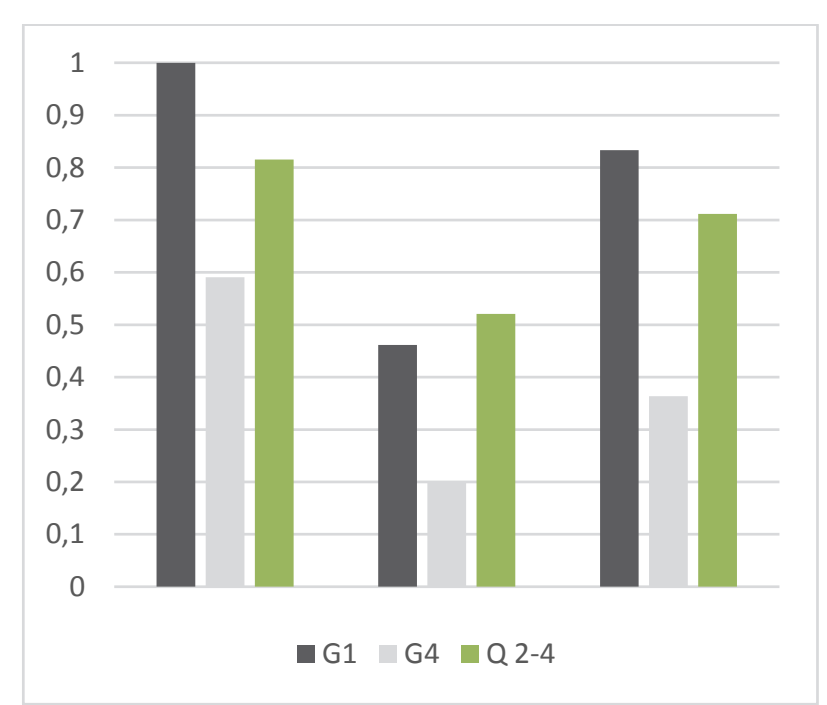

Figura 3. Factores de a) Dedicación y compromiso, b) Asertividad y respeto y c) Motivación, respectivamente, que discriminan entre los G1 y G4 en términos de Huella docente.

Fuente: Los autores.

estudiantes a un proceso profundo de búsqueda intelectual, crecimiento y descubrimiento.

Su constante entusiasmo y motivación, se nota que le gusta

lo que hace $y$ es un sentimiento que transmite a sus estudiantes en cada clase.

Mostrar más interés por su actividad como docente, si no

le gusta enseñar ni le interesa, ese mismo desinterés lo

puedes estar transmitiendo a sus estudiantes.

Cuando las codificaciones realizadas fueron comparadas entre el grupo de mejor (G1) y peor calificación (G4) en la evaluación cuantitativa, se encontró que para huella docente los subfactores que los separaban eran Dedicación y Compromiso, Asertividad, y Motivación y Actitud. Como se puede ver en la Fig. 3, las personas que fueron calificadas más alto en la evaluación cualitativa (G1), también tuvieron una mayor frecuencia de conteos positivos que negativos en estos subfactores.

\section{Conclusiones}

La categorización de las evaluaciones cualitativas permitió identificar categorías emergentes que intervienen en el proceso educativo dentro del aula y que están relacionadas con la percepción que los estudiantes tienen de una clase. Dichas categorías complementan los análisis cuantitativos y proveen información importante que puede permitirles a los docentes reorientar sus actividades de una manera que mejore su desempeño docente. En este sentido, este artículo representa una guía para que los docentes modifiquen sus prácticas para mejorar su desempeño, y sus evaluaciones cuantitativas, cosa que no siempre es evidente para ellos.

En particular, los docentes pueden apoyarse en la literatura psicológica y educativa para mejorar sus limitaciones en cada una de las categorías grandes. En el caso de metodología y sus subfactores es claro que el establecimiento de rutinas (Leinhardt \& Greeno, 1986; Lewis, 2013) puede ayudar a hacer predecible el proceso de enseñanza para los estudiantes, particularmente, en relación con la organización de tiempos y espacios, y la dinámica y participación. En la medida en que el docente genera una secuencia predecible y diversa dentro de su clase, los aspectos tienen una mayor facilidad para concentrar sus recursos cognitivos en el aprendizaje. En ese sentido también es importante señalar que, para mejorar el factor de metodología, los profesores deben ser capaces de combinar tareas abiertas y cerradas, y de establecer estrategias instruccionales particulares para cada una. Si se revisan, por ejemplo, los comentarios sobre acompañamiento y aprendizaje autónomo del estudiante, se puede ver que los estudiantes valoran el aprendizaje autónomo, pero también la guía del docente en ciertas circunstancias. En este sentido, es necesario que los docentes pongan mucha atención a los momentos en los cuales usan estas dos estrategias instruccionales.

Los comentarios de los estudiantes también señalan la necesidad de incorporar problemas o ejemplos transferibles a contextos de actividad real. Esto es palpable, por ejemplo, en los fragmentos referentes a las analogías y las aplicaciones a contextos cotidianos. Lo que esto señala, a un nivel más teórico es la necesidad de incorporar ejemplos y situaciones que tengan transferencia a la práctica profesional. Los estudiantes parecen percibir que la educación de tablero, enfocada en elementos formales, si bien es valiosa, debe ser enmarcada de forma tal que sea evidente su relación con problemas de la práctica profesional. Esto, por supuesto, implica traer elementos isomorfos al dominio de aplicación, y no sólo al espacio académico [18]. Por ejemplo, el álgebra matricial puede presentarse en contextos donde su función fundamental para resolución de sistemas de ecuaciones complejos sea clara para los estudiantes. Esta tarea, aunque implica un trabajo extra, es simple para un investigador o profesional con experiencia en el dominio, y tiene efectos muy fuertes sobre el aprendizaje, transferencia y motivación de los estudiantes.

En relación con la dimensión de dominio y experticia, los resultados señalan que por lo menos para el caso de ingeniería, la percepción que los estudiantes tienen de la competencia del docente puede ubicarse en dos niveles: el nivel de la experiencia y el dominio del tema. En este sentido, sería necesario complementar las teorías tradicionales de experticia [21], para señalar que este constructo es percibido en dos espacios claramente diferenciados: uno asociado al desempeño profesional, y otro asociado a la comprensión del contenido. Una revisión de los comentarios también señala que la experticia propia de los docentes también puede tener efectos adversos sobre el proceso de enseñanza. La literatura psicológica ha señalado que la experticia conlleva una disminución en la capacidad para reconocer y hacer explícitos los procesos subyacentes a su alto desempeño [33]. Este fenómeno, que se ha denominado como el punto ciego del experto, previene a los expertos de identificar sub-secuencias en los procesos de resolución de problemas. En los comentarios, particularmente en aquellos sobre retroalimentación y explicación del tema, se puede ver como los estudiantes piden a los profesores que no den por obvios ciertos temas, y que expliquen los detalles del procedimiento. Esto señala entonces la necesidad de que, en la tarea pedagógica, los docentes, 
expertos en sus áreas, hagan el esfuerzo de hacer explícitos todos los elementos de la tarea. Esta es probablemente la razón por la cual los conteos en dominio y experticia no necesariamente favorecen a los docentes mejor calificados en la evaluación cuantitativa.

En relación con la huella docente es altamente relevante señalar la percepción que los estudiantes tienen del efecto que los docentes tienen sobre la vida de los estudiantes, más allá de su desempeño académico. Este efecto, consistente con la teoría previa [27], se operacionaliza en la percepción de la dedicación y compromiso de los docentes con el aprendizaje del estudiante, lo que constituye un proxy al ejemplo que los docentes proveen en relación con el compromiso con el trabajo y el desempeño profesional. Este efecto se relaciona también con la asertividad, la capacidad de manejar conflictos y de guiar a los estudiantes. En este sentido, el docente es un modelo del tipo de persona que el estudiante debe ser: ¿es alguien respetuoso o por el contrario es agresivo; es amable o antipático? Aunque estos factores parecen secundarios constituyen una parte fundamental de la tarea docente, particularmente en el nivel superior, en el cual las identidades de los estudiantes no están totalmente formadas [34]. Finalmente, este factor se relaciona con la motivación y la actitud que transmite el docente frente a su trabajo: la percepción que los estudiantes tienen de que tanto el docente se entusiasma con su trabajo, con su profesión y con el contenido subyacente. Todos estos elementos importantes en términos de la huella que los docentes dejan a los estudiantes para su vida como profesionales, pero también como personas.

\section{Referencias}

[1] Gallant, A. and Mayer, D., Teacher performance assessment in teacher education: An example in Malaysia, Journal of Education for Teaching, 38(3), pp. 295-307, 2012.

[2] Sulaiman, M., Ismail, Z.H., Aziz, A.A. and Zaharim, A., Lesson study: Assessing pre-service teacher's performance of teaching chemistry, in Engineering Education (ICEED), 2011 3rd Int. Cong., 2011, pp. 208213.

[3] Wei, W., Using summative and formative assessments to evaluate EFL teachers' teaching performance, Assessment \& Evaluation in Higher Education, 40(4), pp. 611-623, 2015. DOI: 10.1080/02602938.2014.939609

[4] Yan, Y., Zhu, Q. and Yang, M., Research on the value-model assessment of performance review for university teachers, in E-Product E-Service and E-Entertainment (ICEEE), 2010 Int. Conf., 2010, pp. 1-4.

[5] Mejía, J.E., Sánchez, P.H. y Visbal, D.A., Evaluación docente mediante BSC y DEA, Revista Educación en Ingeniería, 1(2), pp. 70-86, 2006.

[6] Leinhardt, G. and Greeno, J.G., The cognitive skill of teaching, Journal of Educational Psychology, 78(2), pp. 75-95, 1986. DOI: 10.1037/00220663.78.2.75

[7] Lewis, T., Validating teacher performativity through lifelong schooluniversity collaboration, Educational Philosophy and Theory, 45(10), pp. 1028-1039, 2013. DOI: 10.1111/j.1469-5812.2012.00859.x

[8] Leinhardt, G., Weidman, C. and Hammond, K.M., Introduction and integration of classroom routines by expert teachers, Curriculum Inquiry, 17(2), pp. 135-176, 1987. DOI: 10.1080/03626784.1987.11075284

[9] Leinhardt, G., Math lessons: A contrast of novice and expert competence, Journal for Research in Mathematics Education, 20(1), pp. 52-75, 1987. DOI: $10.2307 / 749098$

[10] Schraw, G., Dunkle, M.E. and Bendixen, L.D., Cognitive processes in well $\square$ defined and ill $\square$ defined problem solving, Applied Cognitive Psychology, 9(6), pp. 523-538, 1995. DOI: 10.1002/acp.2350090605
[11] Langley, P., Pearce, C., Barley, M. and Emery, M., Bounded rationality in problem solving: Guiding search with domain-independent heuristics, Mind \& Society, 13(1), pp. 83-95, 2014. DOI: 10.1007/s11299-0140143-y

[12] Hayes, J.R., The complete problem solver. New York: Routledge, 2013.

[13] Simon, H.A., The structure of ill-structured problems, in Models of Discovery, H.A. Simon, Ed. Berlin, Germany: Springer, 1977, pp. 304325.

[14] Dean Jr, D. and Kuhn, D., Direct instruction vs. discovery: The long view, Science Education, 91(3), pp. 384-397, 2006. DOI: 10.1002/sce.20194

[15] Klahr, D., To everything there is a season, and a time to every purpose under the heavens: What about direct instruction?, in: Constructivist theory applied to instruction: Success or failure?, Tobias, S. and Duffy, T.M. Eds., New York: Taylor and Francis, pp. 291-310. 2009.

[16] Herrington, J., Reeves, T.C. and Oliver, R., Authentic learning environments, in: Handbook of Research on Educational Communications and Technology, Spector, J.M., Merrill, M.D., Elen, J. and Bishop, M.J. Eds., New York: Springer, 2014, pp. 401-412.

[17] Sternberg, R.J., The triarchic mind: A new theory of human intelligence, New York: Viking Pr, 1988.

[18] Kotovsky, K., Hayes, J.R. and Simon, H.A., Why are some problems hard? Evidence from Tower of Hanoi, Cognitive Psychology, 17(2), pp. 248-294, 1985. DOI: 10.1016/0010-0285(85)90009-X

[19] Klahr, D. and Nigam, M., The equivalence of learning paths in early science instruction effects of direct instruction and discovery learning, Psychological Science, 15(10), pp. 661-667, 2004.

[20] Ge, X. and Land, S.M., Scaffolding students' problem-solving processes in an ill-structured task using question prompts and peer interactions, Educational Technology Research and Development, 51(1), pp. 21-38, 2003. DOI: $10.1007 / \mathrm{BF} 02504515$

[21] Ericsson, K.A., Charness, N., Feltovich, P.J. and Hoffman, R., Eds., The Cambridge handbook of expertise and expert performance. Cambridge: Cambridge University Press, 2006.

[22] Ericsson, K.A., Krampe, R.T. and Tesch-Römer, C., The role of deliberate practice in the acquisition of expert performance, Psychological Review, 100(3), pp. 363-406, 1993. DOI: 10.1037/0033295X.100.3.363

[23] Ericsson, K.A. and Smith, J., Toward a general theory of expertise: Prospects and limits, Cambridge: Cambridge University Press, 1991.

[24] Linhares, A. and Brum, P., How can experts see the invisible? Reply to Bilalić and Gobet, Cognitive Science, 33(5), pp. 748-751, 2009. DOI: 10.1111/j.1551-6709.2009.01043.x

[25] Ericsson, K.A., Recent advances in expertise research: A commentary on the contributions to the special issue, Applied Cognitive Psychology, 19(2), pp. 233-241, 2005. DOI: 10.1002/acp.1111

[26] Boaler, J. and Greeno, J.G., Identity, agency and knowing in mathematical worlds, in: Multiple perspectives on mathematics teaching and learning, Boaler, J. Ed., Westport, CT: Ablex, 2009, pp. 171-200.

[27] Vagan, A., Towards a sociocultural perspective on identity formation in education, Mind, Culture, and Activity, 18(1), pp. 43-57, 2011. DOI: 10.1080/10749031003605839

[28] Gresalfi, M., Martin, T., Hand, V. and Greeno, J., Constructing competence: An analysis of student participation in the activity systems of mathematics classrooms, Educational Studies in Mathematics, 70(1), pp. 49-70, 2008. DOI: 10.1007/s10649-008-9141-5

[29] Miles, M.B., Huberman, A.M. and Saldaña, J., Qualitative data analysis: A methods sourcebook. Thousand Oaks, CA: SAGE Publications, 2013.

[30] Namey, E., Guest, G., Thairu, L. and Johnson, L., Data reduction techniques for large qualitative data sets, in: Handbook for teambased qualitative research, Guest, G. and MacQueen, K.M., Eds., Lanham, MD: AltaMira, 2009, pp. 137-161.

[31] Strauss, A.L., Qualitative analysis for social scientists. Cambridge: Cambridge University Press, 1987.

[32] Patton, M.Q., Qualitative evaluation and research methods (2nd Edition). Thousand Oaks, CA: Sage Publications, 2001. 
[33] Nathan M.J. and Petrosino, A., Expert blind spot among preservice teachers, American Educational Research Journal, 40(4), pp. 905-928, 2004.

[34] Landau, M.J., Oyserman, D., Keefer, L.A. and Smith, G.C., The college journey and academic engagement: How metaphor use enhances identity-based motivation, Journal of Personality and Social Psychology, 106(5), pp. 679-698, 2014. DOI: 10.1037/a0036414

Z. Zuluaga-Rendón, es Psicólogo de la Universidad Nacional de Colombia. Ha trabajado en varios proyectos en educación y víctimas del conflicto armado. Actualmente es asistente editorial de la Revista Colombiana de Psicología. Departamento de Psicología Universidad Nacional de Colombia.

ORCID: 0000-0003-0681-4243

J.A. Corredor, es Psicólogo de la Universidad de los Andes y PhD. en Educación de la Universidad de Pittsburg, PA. Es profesor asociado del Departamento de Psicología de la Universidad Nacional de Colombia desde el 2008 en donde ha realizado varios proyectos. Realizó su posdoctorado en Educación con especialidad en videojuegos en la Universidad de Madison, WS. Actualmente se encuentra adscrito al Centro de Innovación Educativa Regional (CIER)-Región Centro, Facultad de Ingeniería, Universidad Nacional de Colombia y al grupo de investigación A1 de Colciencias Cognición y Prácticas de Aprendizaje.

ORCID: 0000-0003-0893-6332

J.M. Quintero, Ph.D., es Ing. de la Universidad Nacional de Colombia. Ha realizado varias especializaciones y obtuvo su doctorado en la Universitat Politècnica de Catalunya. Actualmente es profesor del Departamento de Ingeniería Eléctrica y Electrónica (DIEE) de Universidad Nacional de Colombia, Sede Bogotá, del cual fue director hasta el presente año. ORCID: 0000-0002-3053-0673

J.J Ramirez-Echeverry Ph.D, es Ing. Eléctrico de la Universidad Nacional de Colombia en donde también realizó su MSc. en Ingeniería de Telecomunicaciones. Dentro de sus áreas de interés se encuentran las ciencias educativas por lo que realizó su doctorado en la Universitat Politècnica de Catalunya en Ingeniería de Proyectos y Sistemas. Es profesor del Departamento de Ingeniería Eléctrica y Electrónica (DIEE) Universidad Nacional de Colombia, Sede Bogotá, desde el año 2004.

ORCID: 0000-0002-6499-1785

F.A.ndrés Olarte Ph.D., Ing. Eléctrico de la Universidad Distrital. Obtuvo su MSc. y Dr. en la Universidad Nacional de Colombia, en donde se desempeña como docente desde el año 2010. Actualmente es el director del área curricular del programa de Ingeniería Eléctrica y Electrónica, además de ser el director del Centro de Innovación Educativa Regional (CIER)-Región Centro.

ORCID: 0000-0001-8450-0691 